\title{
Critical Path Minimization Using Retiming and Algebraic Speed-Up
}

\author{
Zia Iqbal $\dagger$, Miodrag Potkonjak $\ddagger$, Sujit Dey $\ddagger$, Alice Parker $\dagger$ \\ †Dept. of EE - Systems, University of Southern California, Los Angeles, CA \\ $\$ C \& C$ Research Laboratories, NEC USA, Princeton, NJ
}

\begin{abstract}
The power of retiming is often limited by the underlying topology of a computational structure. We combine the power of retiming with a complete set of algebraic transformations in an iterative improvement framework, where retiming and algebraic speed-up algorithms are successively applied, so that the latter enables the former. The key part of the approach is a new algebraic speed-up algorithm being used for the first time in high-level synthesis for transformations of algebraic expressions so that an arbitrary set of input arrival times and output required times are satisfied. Since the new method moves delays forward only and retiming is done locally and very infrequently, it also always calculates the new initial state efficiently. The proposed approach has yielded results better or equal to the best previously published on all benchmark examples and on several novel real-life examples.
\end{abstract}

\subsection{Introduction}

Rapid progress of semiconductor processing technology, integrated devices design methodology, and a widespread availability of CAD tools in the last two decades alleviated in many application domains the importance of area as the most critical parameter of a design. This (r)evolution has simultaneously elevated the optimization of throughput (minimum time needed between the acceptance of two successive data samples) to the position of key design parameter. The majority of optimization problems associated with throughput in high-level synthesis are trivial as long as cost is not a factor. For example in scheduling, any scheduling technique which honors the critical path is equally good for this task. However, improvements due to this type of optimization are often not sufficient to meet ever increasing requirements for higher throughput. In this situation, transformations have been widely recognized as an efficient way to provide a competitive advantage in design.

While there is an abundance of published transformation techniques which efficiently trade latency (the time period between arrival of input sample and generation of the output sample) for throughput (most often through pipelining, sometimes compounded with other transformations), in many situations, the real-time nature of an application imposes strict limits on additional latency. In those situations, retiming has proven to be a useful optimization technique to optimize throughput. However, the power of retiming is often limited by the structure of computation. Also, algebraic and redundancy elimination and introduction transformations have been proposed several times, most often in the tree-height reduction framework, as an efficient technique for throughput optimization, without additional latency introduction. However, the power of algebraic and redundancy manipulation transformations is limited by the initial position of delays. Only by targeting both functional restructur- ing and retiming can the maximal throughput be achieved. Figure 1, which shows the simple FIR filter, illustrates this point.
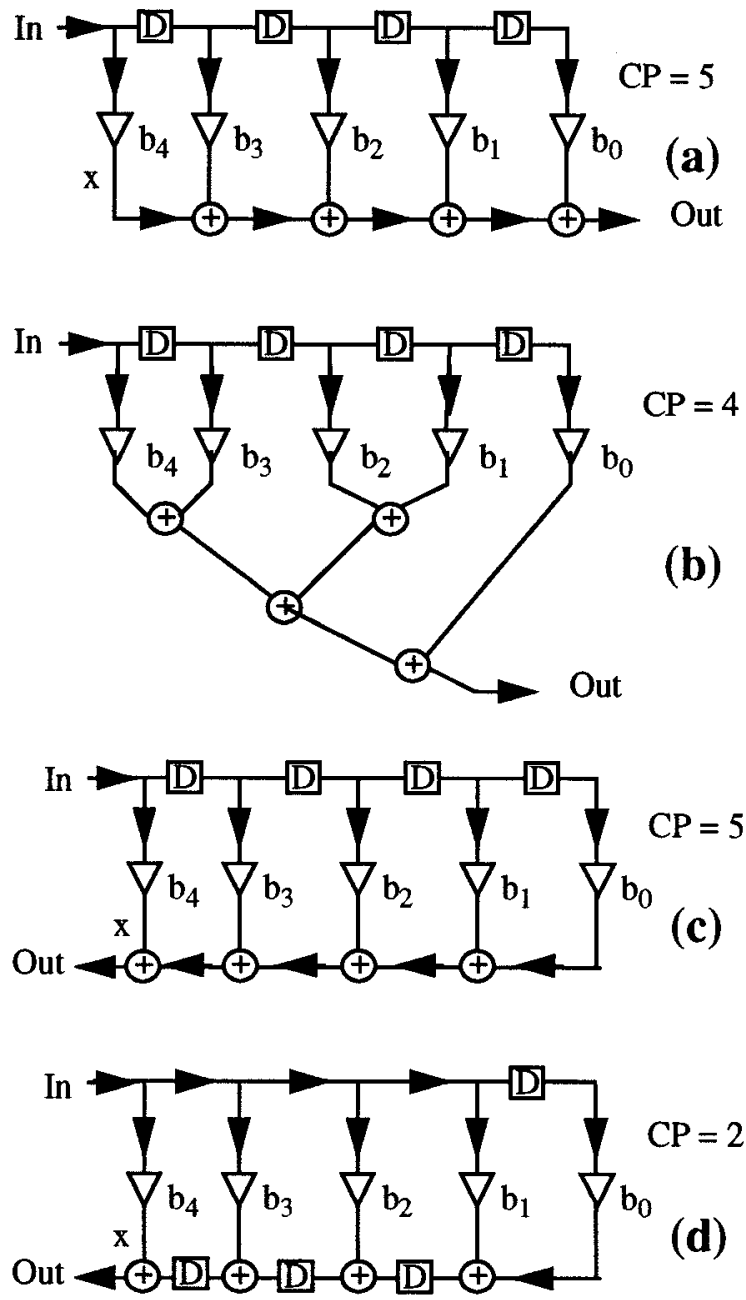

FIGURE 1. The FIR filter example: (a) the initial CDFG; (b) CDFG after application of tree height reduction; (c) after application of associativity; (d) after retiming.

For simplicity, we assume that each operation in the FIR filter example takes one control cycle. The initial critical path is 5 control cycles long (see Figure 1a). It is easy to see that retiming is not effective on this example, and can not reduce the critical path. Associativity and commutativity, as used in several

30th ACM/IEEE Design Automation Conference

Permission to copy without fee all or part of this material is granted provided that the copies are not made or distributed for direct commercial advantage, the ACM copyright notice and the title of the publication and its date appear, and notice is given that copying is by permission of the Association for Computing Machinery. To copy otherwise, or to republish, requires a fee and/or specific permission. 
tree-reduction-based techniques, are slightly more effective: the critical path can be reduced to 4 control cycles, as shown in Figure $1 \mathrm{~b}$.

However, if transformation steps are applied (as shown on Figures 1c and 1d) we can obtain a significantly better reduction in the length of the critical path. The idea is to identify the specific reasons why retiming is ineffective and how the structure of the computation has to be transformed, and then directly target and restructure those places in the control-data flow graph (CDFG) structure, using algebraic transformations, so that retiming for critical path reduction is enabled. As we already mentioned, although there are 4 delays in the FIR filter example, none of them can be used to reduce the critical path. The reason behind this situation is the fact that there is no delay on edge $x$ (Fig 1a), so no delay can be moved across the addition operation to the critical path. In this situation associativity directly helps. If we applied associativity on the chain of additions in the example, the critical path is unchanged, 5 control cycles long. However, now retiming is enabled and it reduces the critical path to only 2 control steps. So algebraic transformations are better utilized in this way than in the tree height minimization framework, although they did not directly reduce the critical path at all.

While the combined application of algebraic transformations and retiming was straightforward in the case of the FIR filter, it is apparent that in general case it poses complex optimization task. This paper presents systematic way for this tasks, by presenting a method for enabling retiming using algebraic transformations by generalizing the ERB [Dey92] approach from logic synthesis research. A key novel component of the approach is the algebraic speed-up technique, which combines the power of all algebraic and redundancy manipulation transformations to maximize the probability that an arbitrary set of required and arrival times are satisfied. By introducing the iterative improvement framework, it also significantly enhances the power of the initial ERB algorithm.

\subsection{Transformations - State of the art}

Recently, transformations have been rapidly establishing themselves as one of the most effective ways to improve a design's parameters. Transformations have been investigated and regularly used for performance enhancement in several engineering areas, most vigorously in software compilers and logic synthesis. Optimizing software compilers usually employ a set of transformations for speed improvement and code size reduction. More recently, compilers for parallel processing systems have been augmented with a set of transformations which explicitly target concurrency exploration. Detailed surveys of most popular transformations in compiler research and engineering can be found in [Fis85].

Transformations in logic synthesis are most often studied in two frameworks, combinational and sequential optimization. Combinational optimization uses algebraic, Boolean and redundancy manipulation technique [Bra90], while sequential optimization augments the set of transformations with retiming. [DeM91, Ma191]. The most elaborate sets of transformations in high level synthesis are given in [Tri87, Wal89, Har89b, Rab91].
Other notable work in this area includes [Gyr84, Goo90, Wo191]. Several in-depth studies of many important aspects of pipelining, an important transformation related to retiming) are also available [Par88, Pau89].

Retiming is a computation technique in which delays are added at some places, and removed from others in such a way that the functionality of the computation is not altered [Lei83]. In high level synthesis, it has been used for the optimization of variety of design goals, including critical path [Har89a]. area [Pot90] and power [Cha92]. Due to their simplicity of application, associativity and commutativity are the two most often applied algebraic transformations in high-level synthesis. Several researchers applied them in the tree height reduction framework [Har89b]. Recently Potkonjak [Pot92] showed an approach which guarantees the optimal application of all algebraic and redundancy manipulation transformations for critical path reduction, but the application domain was restricted to the class of linear computations.

\subsection{Enabling Retiming using Algebraic Transformations}

The ERB (eliminating retiming bottlenecks) method is a technique which enables retiming by changing the computational structure recently introduced and applied in logic synthesis domain [Dey92]. In this paper, we modify and enhance this technique to be applicable to CDFGs. Although there are striking similarities in optimization tasks done in logic synthesis and high-level synthesis domains, there has been surprisingly little effort to explore this relationship by adapting methods of one area and applying it to another. The major reason is that regardless of the mentioned similarities, there are also several sharp differences in computations done in the logic synthesis domain and the high-level synthesis domain, which prevent direct sharing of methodologies and give a different flavor to the problems. Those differences include different delay models and different sets of applicable transformations as described in detail in [Iqb93].

We first briefly review the ERB technique, for a detailed description see [Dey92]. The basic idea of the ERB technique is to identify the bottlenecks which prevent retiming to achieve the desired clock period of a sequential circuit, and eliminate these bottlenecks by satisfying a set of sufficient timing conditions using algebraic transformations. There are three different types of bottlenecks: critical loops and critical combinational and sequential paths. The ERB technique has three steps. In the first step the bottlenecks are translated into sufficient timing conditions. These conditions are satisfied using combinational transformations in the second step. For combinational optimization in addition to SIS speed-up [Sin88] which acts as the optimization engine, a novel cnode cover algorithm is used which judiciously applies common subexpression replication and partitioning to enhance the effectiveness of algebraic and Boolean transformations. The final step is retiming during which all delays are moved only forward so that new shorter critical path is achieved and all new initialization states easily computed. Eliminating the 
bottlenecks ensures that retiming is enabled to achieve the desired clock period.

The different delay models, and in particular the different nature of functional transformations applicable in the two $\mathrm{CAD}$ domains, require substantial modifications and enhancement of the logic synthesis ERB algorithm for application in high- level synthesis. The difference in types of applicable transformations required the development of a new algebraic-speed up technique for CDFGs, which can satisfy given timing constraints. The algebraic speed-up technique, which is described in the next section, in addition to restructuring CDFG using algebraic transformations, checks and ensures during its application that retiming is not disabled due to non-unit delay of various types of operations.

Both the more regular and less involved structure of a CDFG compared to logic circuits make iterative application of the ERB algorithm feasible. Due to regularity, the iterative ERB improved results significantly on all tested examples. Note that the iterative ERB algorithm can be easily adapted to treat a combination of functional pipelining with algebraic and redundancy manipulation transformations also. A simple, well known, formulation of pipelining, that pipelining with $\mathbf{N}$ stages is equivalent to retiming where the number of delays on all inputs is increased by $\mathrm{N}$, directly implies that the only needed modification is to add an appropriate number of delays to all inputs.

\subsection{Algebraic Speed-up}

To enable retiming, the ERB technique requires satisfaction of timing constraints on parts of the CDFG. In this section, we present a new technique which uses an ensemble of several algebraic transformations to satisfy an arbitrary set of timing constraints on the CDFG.

The algebraic speedup technique takes a dataflow graph along with the arrival times for the primary and pseudo-primary inputs, and tries to restructure the computation so as to meet the required time constraints of the outputs. The algorithm can also be used as a critical path reduction technique. In this case the inputs are assigned zero arrival time and the algorithm tries to minimize the critical paths to get the least arrival times at the outputs.

The arrival and required times are assigned by the retiming timing constraints. The critical inputs are those which have to be moved forward during algebraic resynthesis and they are assigned higher (later) arrival times. The other inputs are assigned lower (earlier) arrival times, providing the algorithm freedom for restructuring the CDFG. The common factors in the computation are pushed to a later stage in the computation process so as to make use of common subexpression elimination.

The algorithm begins by constructing an expression tree for the dataflow graph which preserves the order of computations in the dataflow graph. The next step is the extraction of common levels of the computation. Figure $2 \mathrm{a}$ shows the initial and final stages of the CDFG when common level extraction is applied.All the inputs which conform to the associativity laws are identified and gathered under the same parent node. This pro- cess helps to evaluate the level of flexibility in the dataflow graph and the amount of freedom available to schedule the critical inputs.

The next step is the application of distributivity to the dataflow graph, which further increases the chances for common subexpression elimination and the scheduling of the critical inputs. At this stage, we are able to make a trade off between the choice of signals (inputs) for common subexpression elimination and the critical inputs which directly affect the required times of the outputs. The critical signals take preference as they determine the satisfaction of the arrival (required) timing constraints, so in the case of a tie the critical inputs usually get preference. However if the choice of a candidate for common subexpression elimination still enables the timing requirements of the output to be met, then it is chosen, as the process eventually leads to the reduction of operations in the dataflow graph. Figure $2 b$ shows the application of distributivity to the expression tree, which exposes the inputs common to more than one operation nodes.

The common subexpression elimination and factoring process involves the identification of inputs which are common to multiple operations at the same level of computation, and moving them to the next higher level. This process results in a considerable reduction in the number of operations in the CDFG. Consider for example an input which appears in three different operations at the same level of computation. Since each operation will eventually have only two inputs, the identification and subsequent transfer of the input to the next level of computation results in the reduction of instances of the particular operation from three to one. Figure $2 c$ shows the result of common subexpression elimination on the expression tree. Note that the step results in the identification of the input " $e$ " involved with three multiplication operations and its subsequent transformation to reduce it to a single multiplication operation.

After applying level reduction, distributivity and common subexpression elimination, associativity is applied to restructure the CDFG into two-input operations,. Figure $2 \mathrm{~d}$ shows the rearrangement of the expression tree in accordance with the associativity laws so as to meet the arrival time constraints of the inputs. In the case of applying the algorithm to reduce critical paths, this process simply restructures the various inputs at the same level of computation as balanced binary trees so as to minimize their height. In case the goal is to satisfy a set of arrival and required time constraints, the inputs are restructured according to their arrival times: the inputs with earlier arrival times (having maximum freedom) are assigned first and the inputs with the latest arrival times (least freedom) are assigned in the end so as to meet the required time constraints of the output signals.

Illustration of the applications of both algebraic speed-up technique alone and in the iterative ERB framework is presented in [Iqb93a]. Although we concentrated our explanation on the case where only addition and multiplication are used, extension to subtraction and division is simple. While it is straightforward to include subtractions, divisions can be treated using Brent algorithm [Bre74] as a preprocessing step. 

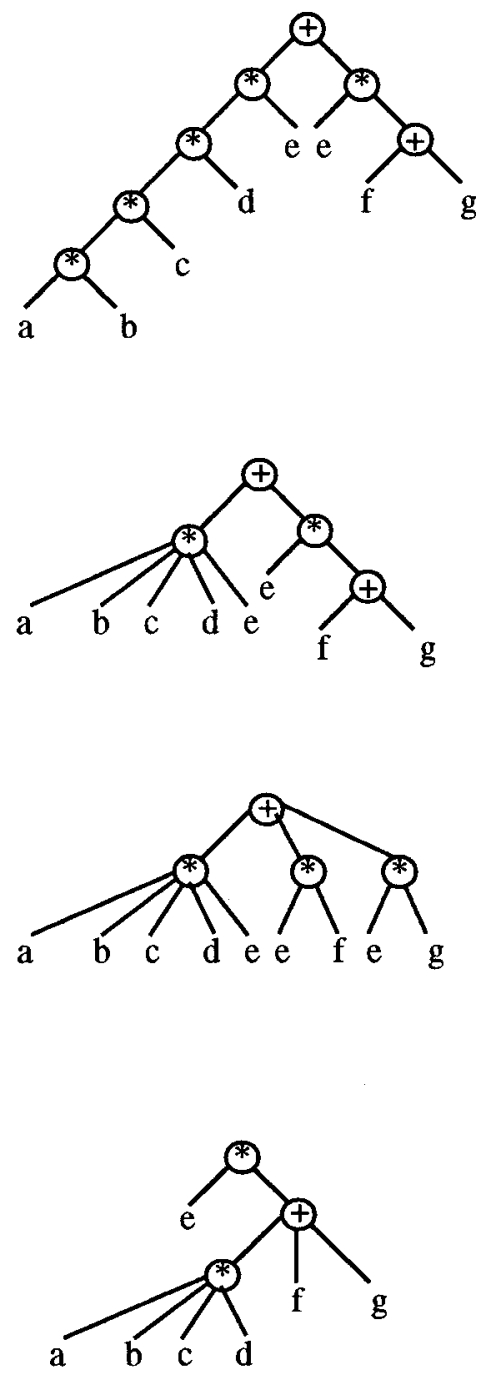

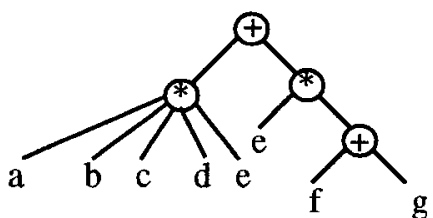

(a)

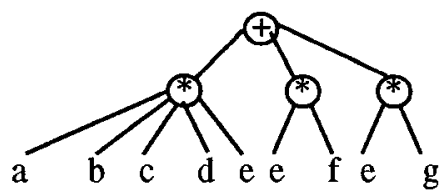

(b)

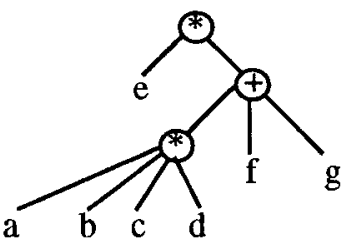

(c)

(d)

FIGURE 2. Intermediate Stages in the Algebraic Restructuring Process: (a) Common Level Extraction, (b) Distributivity, (c) Common Subexpression Elimination and Factoring (d) Associativity.

\subsection{Software Environment and Experimental Results}

Although there is a close technical relationship between logic synthesis and high-level synthesis, software tools used in those two areas have been developed almost without any interaction. Our goal was to leverage as much as possible on it and avoid software rewriting, so we interfaced tools from those two fields.

ERB takes as an input circuits described using the Berkeley Logic Interchange Format (BLIF) and translates it to a data structure like that of the logic synthesis system SIS [Sen92]. Using a cnode cover algorithm with the SIS speed-up algorithm as an engine, ERB provides an ideal software environment for implementation of a single step of our iterative improvement algorithm. We replaced the logic synthesis speed-up algorithm with our new algebraic speed-up code. Since, both logic synthesis and high-level synthesis software use very similar data structures, writing an interface between two data structures was straightforward. As the front end, and for simulation we used the HYPER high-level synthesis system obtained from University of California [Rab91]. Hyper takes as input the behavioral description of a design in a steam-oriented applicative language Silage and translates it to a control data flow graph. We interfaced the CDFG format to the BLIF format.

Table 1 shows a set of 6 examples on which we tested the proposed technique. All examples properly simulate before and after the application of the iterative ERB algorithm. [Iqb93].The average improvement in the length of the critical path in the final design was by a factor of 2.4 times. The average improvement over Leiserson-Saxe retiming is by a factor of 2.16 . The increase 
in the number of operations is rather small, only $22 \%$ for the number of additions and $6 \%$ for the number of multiplications.

The product of the number of operations and the critical path length (NC product), and therefore also AT (area-time) product, are improved simultaneously with the throughput. As shown in Table 1, the NC product is improved on the average by $129 \%$.

\begin{tabular}{|l|c|c|c|c|c|c|c|c|c|c|}
\hline \multirow{2}{*}{ Ex. } & \multicolumn{3}{|c|}{ Initial } & Ret & \multicolumn{3}{c|}{ ERB } & \multicolumn{3}{c|}{ NC Product } \\
\cline { 2 - 11 } & A & M & CP & CP & A & M & CP & I & R & ERB \\
\hline \hline I4 & 8 & 8 & 6 & 4 & 13 & 8 & 3 & 96 & 64 & 63 \\
\hline I4A & 8 & 8 & 6 & 6 & 10 & 8 & 5 & 96 & 96 & 90 \\
\hline LP & 8 & 8 & 9 & 9 & 12 & 12 & 3 & 144 & 144 & 72 \\
\hline V2 & 10 & 17 & 12 & 12 & 10 & 13 & 4 & 324 & 324 & 92 \\
\hline TV2 & 8 & 15 & 10 & 8 & 8 & 13 & 3 & 236 & 184 & 63 \\
\hline A8 & 16 & 13 & 10 & 9 & 16 & 19 & 5 & 290 & 261 & 175 \\
\hline
\end{tabular}

Table 1: Experimental Results: I4- 4th order IIR,; I4A - 4th order adaptive IIR; LP- linear predictor; V2 - 2nd order Volterra Filter, TV2 - 2nd order truncated Volterra filter; A8 - 8th order Avenhaus Filter; A - number of additions, M number of multiplications, CP- critical path, NC - product of number of operations and critical path; I - initial; R - after Retiming; Note that retiming does not change the number of operations

\subsection{Conclusions}

We utilized the ERB sequential performance optimization algorithm to develop an iterative ERB for critical path reduction in high-level synthesis. Experimental results show that the iterative ERB algorithm supported by an algebraic speed-up subroutine outperforms the Leiserson-Saxe retiming algorithm by a factor greater than 2 , with very small increase in hardware requirements, so that on all examples the product of the number of operations and the length of the critical path is improved significantly simultaneously with the throughput. Future work include addressing of other high level synthesis design goals using the ERB technique, and further study of the relationship between high level synthesis and logic synthesis algorithms.

\subsection{References}

[Bre74] R.P. Brent: "The Parallel Evaluation of General Arithmetic Expression", Journal of ACM, Vol. 21, No. 2, pp. 201-206, 1974 .

[Bra90] R. Brayton, et al.,"Multilevel Logic Synthesis", Proc. of the IEEE”, pp. 264-300, Vol. 78, No. 2, Feb. 1990.
[Cha92] A.P. Chandrakasan, at al.: "Hyper-LP: A Design System for Power Minimization using Architectural Transformations", IEEE ICCAD, pp. 300-303, 1992.

[DeM91] G. De Micheli: "Synchronous Logic Synthesis: Algorithms for Cycle-Time Minimization", IEEE Trans. on CAD, Vol. 10, No. 1, pp. 63-73, 1991.

[Dey92] S. Dey, et al.: "Performance Optimization of Sequential Circuits by Eliminating Retiming Bottlenecks", Proceedings of ICCAD-92, pp. 504-509, 1992.

[Fis85] C.N. Fischer, R.J. Le Blank: “Crafting a Compiler”, The Benjamin/Cummings., Menlo Park, CA, 1985.

[Goo90] G. Goossens, et. al. : "An Efficient Microcode Compiler for Application Specific DSP Processors", IEEE Trans. on CAD, Vol. 9, No. 9, pp. 925-937, 1990.

[Gyr84] E. Gyrczyc: "Automatic Generation of Microsequenced Data Paths to Realize ADA Circuit Description", Ph. D. Thesis, Carleton University, 1984.

[Har89a] R. Hartley, A. Casavant: "Tree-height Minimization in Pipelined Architectures", IEEE IC CAD, pp.112-115, 1989.

[Har89b] B.S. Haroun, M.I. Elmasry: "Architectural Synthesis for DSP Silicon Compilers", IEEE Transaction on CAD for IC, Vol. 8, No. 4, pp. 431-447, 1989.

[Iqb93] Z. Iqbal, M. Potkonjak, S. Dey, A. Parker: "Critical Path Minimization Using Retiming and Algebraic SpeedUp", Technical Report \#93-C003-4-5510-1, NEC USA, 1993.

[Lei91] C.E. Leiserson, J.B. Saxe: "Retiming Synchronous Circuitry”, Algorithmica, Vol. 6, pp. 5-35, 1991

[Mal91] S. Malik, et al.: "Retiming and Resynthesis: Optimizing Sequential Networks with Combinatorial Techniques", IEEE Trans. on CAD, Vol. 10, No. 1, pp. 74-84, 1991.

[McF83] M.C. McFarland, A.C. Parker: "An Abstract Model of Behavior for Hardware Descriptions", IEEE Transaction on Computers, Vol. 32, No. 7, pp. 621-636, 1983.

[Par88] N. Park, A.C. Parker: "Sehwa: A Software Package for Synthesis of Pipelines from Behavioral Specifications", IEEE Trans. on CAD, Vol 7, No. 3, pp. 356-370, 1988.

[Pau89] P.G. Paulin, J.P. Knight: "Force -Directed Scheduling for the Behavioral Synthesis of ASIC", IEEE Trans. on CAD, Vol 8., No 6, pp. 661-679, 1989.

[Pot90] M. Potkonjak and J. Rabaey, "Retiming for Scheduling", VLSI Signal Processing, pp. 23-32, San Diego, Nov. 1990.

[Pot92] M. Potkonjak, J. Rabaey: "Maximally Fast and Arbitrarily Fast Implementation of Linear Computations", IEEE ICCAD, pp.304-308, November 1992.

[Rab91] J. Rabaey, C. Chu, P. Hoang, M. Potkonjak: "Fast Prototyping of Data Path Intensive Architecture", IEEE Design and Test, Vol. 8, No. 2, pp. 40-51, 1991.

[Sin88] K. J. Singh, et. al.: "Timing Optimization of Combinational Logic", IEEE ICCAD, pp. 282-285, 1988.

[Tri87] H. Trickey: "Flamel: A high-Level Hardware Compiler", IEEE Trans. on CAD, Vol. 6, No. 2, pp. 259-269, 1987.

[Wal89] R.A. Walker, D.E. Thomas: "Behavioral Transformation for Algorithmic Level IC Design" , IEEE Trans. on CAD, Vol 8. No.10, pp. 1115-1127, 1989. 